\title{
Dark Photon Studies at BABAR
}

\author{
Alessandra Filippi ${ }^{1, \star}$ \\ on behalf of the BABAR Collaboration \\ ${ }^{1}$ I.N.F.N. sez. di Torino \\ via P. Giuria, 1, 10125 Torino, Italy
}

\begin{abstract}
An overview of the most recent results by $B A B A R$ on the search of dark photon and dark sector gauge bosons is presented, with larger emphasis on dark photon decays in invisible channels and searches for muonic dark force.
\end{abstract}

\section{Introduction}

After more than 80 years since its first postulation by Zwicky, the existence of Dark Matter (DM) can now be considered as consolidated, on account of numerous astrophysical pieces of evidence, all based on the gravitational interaction. However, its nature is nowadays still a mystery. One appealing possibility is that it could be formed by heavy massive particles which can interact only weakly with ordinary matter (the so-called WIMPs). However, this paradigm suffers for the absence of any observation, so far, in direct detection and high energy colliders experiments.

One alternative idea is that beyond Standard Model (SM) Dark Sectors exist, which can host a variety of new particles, that interact with ordinary matter in a feeble way only through gauge mediators via their kinetic couplings. The simplest realization of such sectors would be a $\mathrm{U}(1)_{D A R K}$ symmetry, matched to the hypercharge U(1) SM one, whose gauge carrier is kinetically coupled to the photon and therefore to SM fermion pairs, through a small coupling $\epsilon_{Y}$. The $\mathrm{U}(1)_{D A R K}$ gauge mediator gets a mass in the $\mathrm{MeV}-\mathrm{GeV}$ scale due to electroweak symmetry breaking, and then it could be observable; on the other hand, the kinetic mixing is typically expected to be in the range $10^{-5}-10^{-2}$, but could even be smaller, which accounts for the weakness of the dark boson interaction with ordinary matter.

So far, no evidence of Dark Sector carriers has been found as well. However, a number of experimental observations from terrestrial as well as satellite searches exists, which can be accounted for by Dark Sectors models in a straightforward way. Among them, Dark Sectors could provide an elegant way to explain the apparent discrepancy of the observed $(g-2)$ value of the muon [1]. Moreover, they can explain the large positron/electron yields excess, without a corresponding counterpart in antiproton/proton, as reported by several experiments (such as HEAT, PAMELA, PPB-BETS, ATIC and AMS), the $511 \mathrm{KeV}$ line observed from the galactic center observed by INTEGRAL and also the DAMA/LIBRA modulation effect [2].

If the gauge mediator is a spin-1 particle (in this case the sector is also named "vector" portal), a natural place to look for a dark, massive photon $A^{\prime}$ is represented by $e^{+} e^{-}$factories. The cross-section

\footnotetext{
^e-mail: filippi@to.infn.it
} 
for its production could be as large as some tenths of $\mathrm{fb}$, and the sensitivity for such a signal would be proportional to the integrated luminosity (but inversely proportional to the available center-of-mass energy).

The ways in which such particles could be observed depend on their mass and coupling, also relative to other DM states belonging to the same Dark Sector. In general, dark photons can always appear whenever SM photons are produced, with an additional $\epsilon_{Y}^{2}$ suppression factor. As the lightest particle of the Dark Sector, the dark photon is expected to decay to di-leptons or light di-hadron pairs, and it could be observed as a narrow resonance $\left(\Gamma \sim m \epsilon_{Y}^{2}\right)$ in the invariant mass spectrum of the pair. If, on the other hand, some lighter and stable enough DM states exist $(\chi)$, one would expect the dark photon to decay to invisible channels. These decays would be dominating as not $\epsilon_{Y}$ suppressed. Were the $\chi$ states unstable, one would expect more complicated signatures in which many leptons could appear, as a consequence of off-shell patterns including also Higgs and heavy leptons and quarks loops.

\section{Search for Dark Photon visible decays at BABAR}

$B A B A R$ [4] was one of the first experiments where searches of dark photon were performed. In its 10 years life (with the last data-taking in 2008), BABAR collected more than $500 \mathrm{fb}^{-1}$ of $e^{+} e^{-}$annihilation events, with beams delivered by the PEP-II machine at SLAC at several energies corresponding to $\Upsilon(2,3,4 \mathrm{~S})$ bottomonium excitations. Even though the integrated luminosity is going to be overtaken soon by the upcoming BELLE-II experiment, the $\Upsilon(2 S)$ sample collected by BABAR will remain the richest ever for many years to come.

The first results on dark photon searches by BABAR were published in a well-known paper in 2014 [5], in which the study of a dark photon production in the $e^{+} e^{-} \rightarrow \gamma A^{\prime}$ radiative return reaction and its following decay in $e^{+} e^{-}$or $\mu^{+} \mu^{-}$pair was presented. This analysis went along in parallel with searches of new (beyond SM) pseudoscalar particles - in particular belonging to the light Higgs sector, produced by $e^{+} e^{-} \rightarrow h A^{\prime}$ Higgs-strahlung followed by light Higgs decays into a dark photon pair $h \rightarrow A^{\prime} A^{\prime} \rightarrow\left(\ell^{+} \ell^{-}\right)\left(\ell^{\prime+} \ell^{\prime-}\right)$ [6], as well as dark gauge bosons produced in dark photon decays $\left(e^{+} e^{-} \rightarrow \gamma A^{\prime}, A^{\prime} \rightarrow W^{\prime} W^{\prime \prime} \rightarrow\left(\ell^{+} \ell^{-}\right)\left(\ell^{\prime+} \ell^{\prime-}\right)\right)[7]$.

No observation was reported for dark photons neither for other new "exotic" objects; however, $B A B A R$ could provide a wide exclusion area in the $\left(m_{A^{\prime}}, \epsilon_{Y}\right)$ Dark Sector parameters plane, shown in Fig. 1 along with some other regions excluded by other experiments.

The largest local significance for a possible dark photon signal was found to be $3.4 \sigma$ at $7.02 \mathrm{GeV}$ for decays in the $e^{+} e^{-}$channel, and $2.9 \sigma$ at $6.09 \mathrm{GeV}$ for the $\mu^{+} \mu^{-}$one, both being not enough to claim its presence. Together with some more recent results by NA48/2 [8], the BABAR measurement almost excludes the parameter region which would favor a vector Dark Sector as an explanation for the observed $(g-2)_{\mu}$ discrepancy.

\section{Search for Dark Photon invisible decays at BABAR}

The signature for such events, in which the dark photon decays into a pair of light DM $\chi$ particles which go undetected, is just a single photon, produced in the $e^{+} e^{-} \rightarrow \gamma A^{\prime}$ reaction [9]. This involves critical experimental issues in requiring an efficient trigger on single photons and a large hermeticity of the detector, to avoid as much as possible acceptance holes in order to limit the contribution of overwhelming background sources with $\gamma$ 's which can be lost or convert in the detector materials (mainly, $2 \gamma$ and radiative Bhabha events). As far as the trigger is concerned, BABAR could collect about $53 \mathrm{fb}^{-1}$ of single photon events in the last part of its running period (mostly at $\Upsilon(2 \mathrm{~S})$ and $\Upsilon(3 \mathrm{~S})$ 


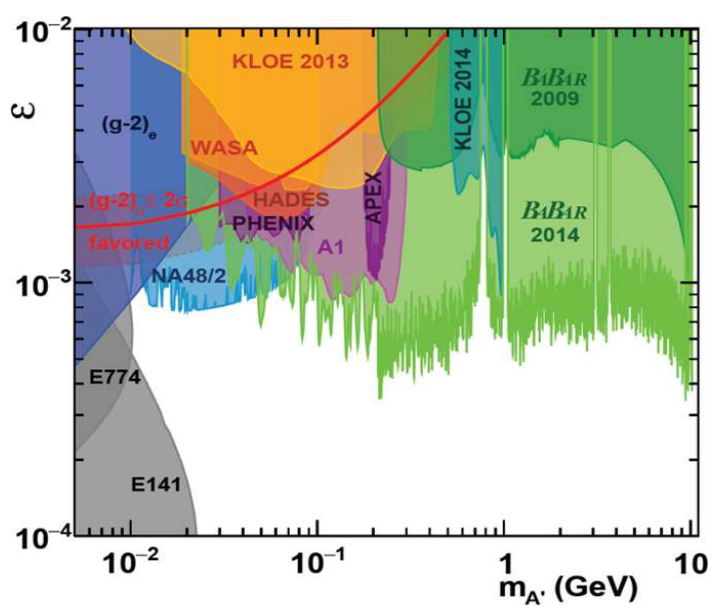

Figure 1. Compilation of existing dark photon constraints in the parameter space $\epsilon_{Y}$ vs dark photon mass, for decays in light lepton pairs.

energies), in which a first level hardware trigger was applied requiring at least one cluster in the electromagnetic calorimeter associated to a photon with energy larger than $800 \mathrm{MeV}$. At third level, two software trigger lines were set-up which distributed the total sample in two subsets according to the center-of-mass photon energy: a high energy sample $\left(E_{\gamma}^{\star}>2 \mathrm{GeV}\right)$, corresponding to a low mass recoiling $A^{\prime}\left(-4<m_{A^{\prime}}^{2}<36 \mathrm{GeV}^{2}\right)$, and a low energy one $\left(E_{\gamma}^{\star}>1 \mathrm{GeV}\right)$, corresponding to a higher $A^{\prime}$ mass region, $24<m_{A^{\prime}}^{2}<69 \mathrm{GeV}^{2}$. In both cases events with tracks coming from the interaction vertex were not accepted. The two samples needed a separate treatment due to a different nature of the background in the two energy regions, and the search of dark photon was performed independently in each of them. Some common criteria were however applied to ensure the detection of a good quality photon well contained in the detector acceptance, without extra charged tracks in the event and very limited electromagnetic noise in the calorimeter. The missing 4-momentum vector, which corresponds to the undetected $A^{\prime}$, was also constrained by tight fiducial cuts in order to prevent leakage effects and conversions, for instance on the electromagnetic calorimeter crystal edges. The external $B A B A R$ muon detector information was also exploited as a veto for events in which conversions could have occurred. Machine learning techniques (Boosted Decision Trees, BDT's) were applied to effectively separate signal from background events.

The search for dark photons proceeds through a scan of the missing mass $\left(m_{X}\right)$ plot in sliding windows, with a width multiple (of the order of $20 \times$ ) of the photon resolution: in each mass slice the spectrum is fitted by a Crystal Ball function to reproduce the possible signal, plus a background function whose shape changes according to the selected data set. In the low mass sample, the main source of background is represented by $2 \gamma$ events in which one of the photons is lost, and its contribution peaks at $m_{X}=0$; in addition to this, a smooth contribution develops at higher masses, produced by radiative Bhabha events. On the other hand, the peaking contribution is missing in the higher mass selection, where only a tail of the $2 \gamma$ reaction contributes while the largest part of the background comes from radiative Bhabha events where the $e^{+} e^{-}$pair is lost due the detector acceptance.

Suitable fits were performed simultaneously on samples taken at different center-of-mass energies, with proper selections on the BDT discriminant controlling the amount of background in each mass slice, for low and high mass samples; fits on control samples populated mainly by background events 
allowed the shape of the background contribution to be fixed and used with constant parameters in addition to the possible resonant signal. The local significance of the signal was estimated through the likelihood ratio $\left.\mathcal{S}=\operatorname{sign}\left(N_{\text {sig }}\right) \sqrt{2 \log \left(\mathcal{L} / \mathcal{L}_{0}\right.}\right)$ in which $N_{\text {sig }}$ corresponds to the integral of the Crystal Ball function used to reproduce the signal contribution, after background subtraction, and $\mathcal{L}$ and $\mathcal{L}_{0}$ are the likelihoods of the fits with and without the inclusion of the resonant signal, respectively. The global maximum significance, extended over all the analysed samples, did not exceed $2.6 \sigma$ (local maximum at $6.21 \mathrm{GeV}$ ), which is not enough to claim for any new structure. However, the evaluation of the upper limit for the production cross section at $90 \%$ C.L. could be performed, and from this the exclusion regions in the dark photon parameter space could be extracted. The areas excluded by $B A B A R$ are shown in Fig. 2: the full band of parameters explaining the $(g-2)_{\mu}$ anomaly as a consequence of the existence of the dark photon is ruled out, down to dark photon masses on the order of the MeV.

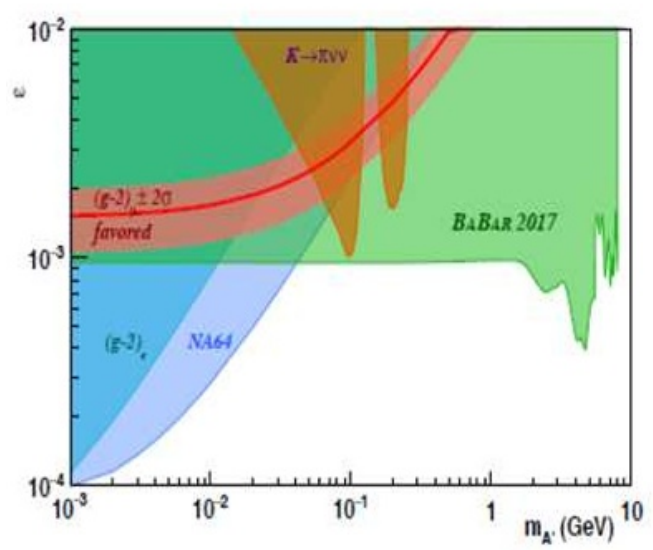

Figure 2. Regions of $A^{\prime}$ parameter space ( $\epsilon_{Y}$ vs $m_{A^{\prime}}$ ) excluded by BABAR analysis [9] of invisible decays, superimposed to previous constraints and the band preferred at $2 \sigma$ for the explananation of the $(g-2)_{\mu}$ anomaly.

\section{Search for Muonic Dark forces}

An alternative search for a Dark Sector vector mediators which could be the carriers of a new symmetry favoring the interaction between heavy leptons $\left(\mathrm{L}_{\mu}-\mathrm{L}_{\tau}\right.$ gauge interaction) was performed by $B A B A R$ looking at multi-muonic final states [10]. These could be reached through the reactions $e^{+} e^{-} \rightarrow \mu^{+} \mu^{-} Z^{\prime}, Z^{\prime} \rightarrow \mu^{+} \mu^{-}$, in which the $Z^{\prime}$ vector gauge boson is produced via radiation from muons (or taus), and subsequently decays to a $\mu^{+} \mu^{-}$pair. Such a model [11] could explain several observed anomalies: beyond the already mentioned $(g-2)_{\mu}$ one, the relative abundance on SM neutrinos compared to sterile ones, and the proton radius discrepancy puzzle.

No observation of the $Z^{\prime}$ gauge boson has been made so far by experiments studying the inelastic interation of neutrinos with nuclei. Given the relatively small mass expected for $Z^{\prime}[12], e^{+} e^{-}$annihilation could again be a suitable environment where to look for its signature: $B A B A R$ for the first time performed its search.

The sample studied by BABAR included the full statistics, collected at all energies of the bottomonium peaks as well as off-peak $\left(514 \mathrm{fb}^{-1}\right)$ : events with four reconstructed muons carrying the full available energy were selected. The extra energy per event, carried by additional neutral particles 
and measured by the electromagnetic calorimeter, was required to be smaller than $200 \mathrm{MeV}$; muons were identified in pairs of the same charge. Events coming from the decay of $\Upsilon(3,2 S)$ to $\Upsilon(1 S) \pi^{+} \pi^{-}$ with the $\Upsilon(1 \mathrm{~S})$ subsequent decay in $\mu^{+} \mu^{-}$and $\pi / \mu$ misidentification were removed from the analysed sample. The invariant mass of the four muon system was studied in a $500 \mathrm{MeV}$ wide window of the nominal center-of-mass energy: the main contribution to this region is given by $4 \mu$ QED background events.

To look for a possible signal in the $\mu^{+} \mu^{-}$invariant mass system, the reduced invariant mass $m_{R}=$ $\sqrt{m_{\mu^{+} \mu^{-}}^{2}-4 m_{\mu}^{2}}$ was studied, in 50 $\sigma$ wide sliding mass windows. Corrections had to be applied since the Montecarlo overestimated, in general, the QED background contribution, as a consequence of an imprecise treatment of ISR photon emission; some additional tracking and PID inefficiences had to be taken into account as well. As in the previous case, the search for a resonant signal in the $\mu^{+} \mu^{-}$ system was performed separately in data samples taken at different energies, and the combination of fit likelihoods was performed only at a final stage.

No significant signal was found in the mass range $0.212<m_{Z^{\prime}}<10 \mathrm{GeV}$, the maximum global significance from the likelihood ratio being just $1.6 \sigma$, with a local maximum of $4.3 \sigma$ at $830 \mathrm{MeV}$.

Again, it was however possible to derive the upper limit for the $Z^{\prime}$ production cross section and decay at $90 \%$ C.L., and from it to deduce the exclusion area in the $\left(m_{Z^{\prime}}, g^{\prime}\right)$ parameter space $\left(g^{\prime}\right.$ being the coupling constant to SM of this new gauge symmetry). Fig. 3 reports the excluded area by BABAR superimposed to those from other neutrino experiments.

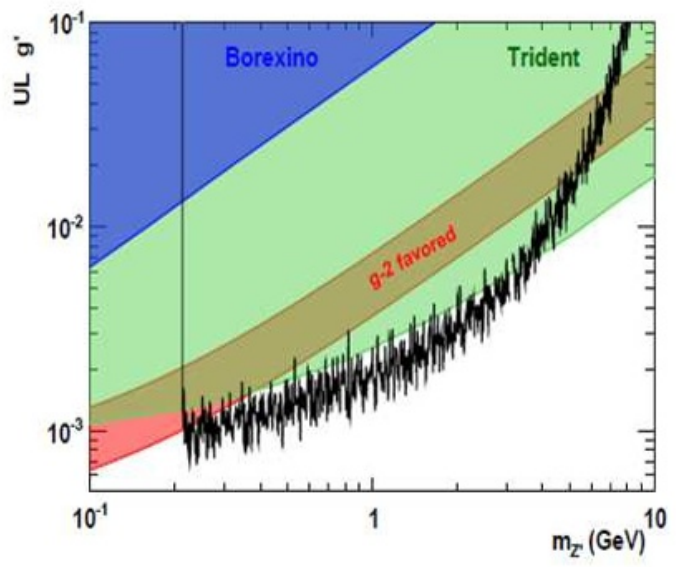

Figure 3. The $90 \%$ upper limit of the gauge coupling $g^{\prime}$ as a function of the $Z^{\prime}$ mass from $B A B A R$ [10], together with the constraints coming from the production of $\mu^{+} \mu^{-}$pairs in $v_{\mu}$ scattering experiments.

Compared to neutrino experiments' results, $B A B A R$ is able to provide more extended exclusion ranges and fixes an upper limit for the $g^{\prime}$ coupling down to $\sim 7 \times 10^{-4}$ close to the dimuon threshold. Apart from a small mass region down a few $\mathrm{MeV}, B A B A R$ is providing these powerful constraints under the hypothesis that the $Z^{\prime}$ gauge boson couples universally with muons and taus and their respective neutrinos, and it rules out almost completely the favoured band for the explanation of the $(g-2)_{\mu}$ anomaly by means of this portal. 


\section{Conclusions}

A broad interest has arisen in the last years about the search for possible light Dark Matter candidates; among them, spin-1 gauge bosons are the most sought, as $e^{+} e^{-}$factories are the ideal places to study their production and decays, together with electron induced interactions at high intensity electron beam facilities [13].

So far, many experiments provided upper limits on their observations and excluded wide regions in the Dark Sector models parameters, due to no evidence of signal. The precision and quality of these measurement will be soon overtaken by the results with high statistics from Belle and the upcoming Belle-II experiments; however, among all experiments at colliders, the BABAR measurements are so far providing the best sensitivity for light Dark Matter searches. Moreover, the BABAR results are able to rule out almost completely the $(g-2)_{\mu}$ favored region, as well as the region of mass down to $1 \mathrm{MeV}$ for dark photons decaying into the invisible channel.

The field is nowadays very lively, with several analyses of $B A B A R$ data still ongoing on the subject: more novel results are expected soon.

\section{References}

[1] M. Pospelov, Phys. Rev. D90 (2009), 095002

[2] B. Batell et al., Phys. Rev. D79 (2009), 115008 and references therein

[3] R. Essig et al., Phys. Rev. D80 (2009), 015003

[4] BABAR Collaboration, B. Aubert et al., Nucl. Instr. Methods A479 (2002), 1

BABAR Collaboration, B. Aubert et al., Nucl. Instr. Methods A729 (2015), 2013

[5] BABAR Collaboration, J.P. Lees et al., Phys. Rev. Lett. 113 (2014), 201801

[6] BABAR Collaboration, B. Aubert et al., arXiv:0808.0018 [hep-ex], interpreted as limits on dark photon parameters by R. Essig et al., in arXiv:1311.0029 [hep-ph]

BABAR Collaboration, J.P. Lees et al., Phys. Rev. Lett. 113 (2012), 211801

[7] BABAR Collaboration, B. Aubert et al., arXiv: 0908.2821 [hep-ex]

[8] NA48/2 Collaboratin, J.R. Batley et al., Phys. Lett. B769 (2017), 67

[9] BABAR Collaboration, J.P. Lees et al., arXiv: 1702.0332 [hep-ex], submitted to PRL

[10] BABAR Collaboration, J.P. Lees et al., Phys. Rev. D94 (2016), 0111012

[11] B. Batell et al., Phys. Rev. Lett. 107 (2011), 011803

W. Altmannhofer et al., Phys. Rev. Lett. 113 (2014), 091801

A. Kamada et al., Phys. Rev. D92 (2015), 113004

[12] B. Shuve, I. Yavin, Phys. Rev. D89 (2014). 113004

[13] See, for instance, J. Alexander et al., arXiv:1608.8632 [hep-ph] 